\title{
Avaliação retrospectiva de artrodese cervical com enxerto autólogo versus hidroxiapatita
}

\author{
Retrospective study of cervical arthrodesis with autograft versus \\ hydroxyapatite graft
}

\section{Estudio retrospectivo de la artrodesis cervical con autoinjerto versus hidroxiapatita}

\author{
Yoshinobu Nagasse' \\ Clóvis Yamazato \\ Fábio Mastromauro Oliveira² \\ lberê Ribeiro² \\ José Olympio Catão Bastos Junior ${ }^{2}$ \\ René Kusabara ${ }^{3}$
}

\section{RESUMO}

Objetivo: os autores realizaram um estudo e compararam o resultado radiográfico no uso de hidroxiapatita e enxerto de crista ilíaca (autólogo) no tratamento cirúrgico de hérnia discal cervical em um nível, sendo realizada artrodese cervical anterior com placa. Métodos: foi realizado estudo retrospectivo com pacientes submetidos à artrodese cervical comparando-se dois grupos de dez pacientes: um em que foi utilizado enxerto de hidroxiapatita e outro em que foi utilizado enxerto de crista ilíaca (autólogo). Radiografias pós-operatórias foram mensuradas e comparadas, à busca de perdas de alinhamento angular. Resultados: os dois tipos de enxerto demonstraram manter alinhamento em taxas similares, sendo observada consolidação óssea em todos os pacientes. O estudo não mostrou fragmentação do enxerto de hidroxiapatita ou soltura do mate-

\section{ABSTRACT}

Objective: the authors carried out a study to compare the radiografic result in the use of hydroxyapatite graft and iliac crest bone graft (autologous) in the surgical treatment of cervical discal herniation in one level, and a cervical arthrodesis was carried out previously with plate. Methods: a retrospective study with patients who were submitted to cervical artrodesis, comparing two groups of ten patients: one in which the hydroxyapatite graft was used and the other in which the autologous iliac crestwas used. Postoperative x-rays were measured and compared, in search for losses of angular alignment. Results: the two types of grafts demonstrated an alignment in similar rates, with consolidation in all patients. The study did not show fragmentation of the hydroxyapatite graft or acquittal of the synthesis material.
\end{abstract}

\begin{abstract}
RESUMEN
Objetivos: los autores realizaram un estudio y compararon el resultado radiográfico en el uso del hidroxiapatita y injerto de cresta ilíaca (autólogo), en el tratamiento quirúrgico de hernia discal cervical en un nivel, siendo realizada artrodesis cervical anterior con placa. Métodos: fue realizado un estudio retrospectivo con pacientes, que habian sido sometidos a la artrodesis cervical con injerto de hidroxiapatita o injerto de cresta iliaca b.z autologa. Las radiografias postoperatorias habian sido mensuradas y comparadas, buscando para las pérdidas de alineación angular. Resultados: los dos tipos de injerto habian demostrado manutención de alineación similares, donde la consolidación fue observada en todos los pacientes. El estudio no demostró la fragmentación del injerto de hidroxiapatita o de soltar el material de
\end{abstract}

\footnotetext{
Trabalho realizado no Grupo de Coluna do Hospital Municipal Dr. Cármino Caricchio - São Paulo (SP), Brasil; Hospital IFOR Ortopedia e Traumatologia - São Bernardo do Campo (SP), Brasil; Hospital IGESP - São Paulo (SP), Brasil.

'Estagiário do Grupo de Coluna do Hospital Municipal Dr. Cármino Caricchio - São Paulo (SP), Brasil; Hospital IFOR Ortopedia e Traumatologia - São Bernardo do Campo (SP), Brasil; Hospital IGESP - São Paulo (SP), Brasil.

${ }^{2}$ Assistente do Grupo de Coluna do Hospital Municipal Dr. Cármino Caricchio - São Paulo (SP), Brasil; Hospital IFOR Ortopedia e Traumatologia - São Bernardo do Campo (SP), Brasil; Hospital IGESP - São Paulo (SP), Brasil.

${ }^{3}$ Chefe do Grupo de Coluna do Hospital Municipal Dr. Cármino Caricchio - São Paulo (SP), Brasil; Hospital IFOR Ortopedia e Traumatologia - São Bernardo do Campo (SP), Brasil; Hospital IGESP - São Paulo (SP), Brasil.
} 
rial de síntese. Conclusão: nesta pequena série de casos não foi observada diferença significativa entre os resultados da artrodese cervical anterior com enxerto de hidroxiapatita em relação ao enxerto autólogo de ilíaco.

DESCRITORES: Fusão vertebral; Hidroxiapatita, Transplante ósseo; Vértebra cervical/ cirurgia; Artrodese/ instrumentação
Conclusion: in this small series of cases, no significant difference was observed between the results of the anterior cervical arthrodesis with hydroxyapatite graft in relation to the iliac crest bone graft.

KEYWORDS: Spinal fusion;

Hydroxyapatite; Bone

transplantation; Cervical

vertebrae/surgery;

Arthrodesis/instrumentation síntesis. Conclusión: en esta pequeña serie de casos no hubo diferencias significativas entre los resultados de la fusión cervical anterior con injerto de hidroxiapatita en comparación con injerto autólogo ilíaca.

\section{INTRODUÇÃO}

$\mathrm{O}$ tratamento das doenças degenerativas cervicais, quando cirúrgico, pode ser realizado por meio da artrodese cervical via anterior com placa $^{1-3}$. O uso da placa ganhou espaço devido à estabilidade precoce, à prevenção da migração de enxerto e à reabilitação precoce ${ }^{1-3}$. A fusão intervertebral permite uma estabilidade sem dependência de instrumental ${ }^{1-3}$. A realização da fusão intervertebral, mantendo o alinhamento e a altura do espaço discal, requer uso de enxerto, geralmente autólogo, retirado da crista ilíaca.

Entretanto, a técnica de extração do enxerto apresenta taxas acima de $20 \%$ de complicação, como infecções, fraturas de pelve, parestesias e dores crônicas ${ }^{4-6}$. As complicações estimulam a busca por alternativas que sejam amplamente disponíveis e que possuam taxas de sucesso semelhantes à artrodese com enxerto autólogo.

A hidroxiapatita (HA) é um tipo de enxerto do grupo das biocerâmicas. É biocompatível, osteocondutor e bioativo $^{7,8}$. A bioatividade ocorre devido às similaridades químicas da HA em relação à parte mineral óssea, permitindo ligações químicas ${ }^{7}$. A osteocondutividade representa a capacidade do enxerto de direcionar a neoformação óssea, por ser um leito favorável para esse processo.

Por causa dessas características, a HA é cogitada como um possível substituto do enxerto ${ }^{6,9,10}$. Além disso, é um produto de menor custo em relação à proteína morfogênica óssea (BMP) e outros aloenxertos.

\section{MÉTODOS}

Neste estudo retrospectivo, foram avaliados 20 pacientes submetidos à artrodese cervical anterior com placa e enxerto autólogo bicortical ou bloco compacto de hidroxiapatita. Todos os pacientes apresentavam hérnia cervical em um nível e todos estavam acometidos por cervicobraquialgia, sem resposta ao tratamento farmacológico e fisioterápico por no mínimo três meses. Os pacientes foram acompanhados de um a três anos (média de dois anos).

Foram feitas avaliações radiográficas no pré, no pósoperatório imediato e no acompanhamento ambulatorial.
As radiografias foram avaliadas buscando em busca de evidências de formação de pontes ósseas para confirmação de artrodese. Evidências de não consolidação foram consideradas como perda de alinhamento maior que $3^{\circ} \mathrm{e}$ soltura de material sem ponte óssea.

\section{TÉCNICA CIRÚRGICA}

Os pacientes foram posicionados em decúbito dorsal, e a incisão sobre a prega cutânea foi realizada de acordo com o nível a ser abordado. A abordagem de escolha foi à esquerda.

Após a realização do acesso às vértebras cervicais ${ }^{11}$, confirmou-se o nível a ser abordado com auxílio de fluoroscopia. A discectomia foi feita com curetas e pinças de disco e realizou-se uma leve distração com o afastador de Caspar.

A extração de enxerto ilíaco foi feita através de uma incisão longitudinal à crista ilíaca. Obtivemos enxerto bicortical da tábua interna por preservar o relevo da crista ilíaca, diminuindo também a dor pós-operatória.

O enxerto de hidroxiapatita, em formato de bloco, foi escolhido dependendo do espaço a ser preenchido.

Após o posicionamento do enxerto, implantou-se a placa com os parafusos, sendo realizada a sutura por planos.

\section{RESULTADOS}

Os pacientes foram avaliados por radiografias no pré-operatório, no pós-operatório imediato e nosacompanhamentos ambulatoriais (Tabela 1). Foram medidos os ângulos complementares entre o platô superior da vértebra superior e o platô inferior da vértebra inferior nas radiografias em perfil de todos os acompanhamentos pós-operatórios, com o objetivo de averiguar progressão de desvio.

Foram considerados com desvio os casos que evoluíram com diferença de $3^{\circ}$ ou mais em seu acompanhamento, em relação à radiografia pós-operatória imediata.

Nos dez casos operados com enxerto de hidroxiapatita (Figura 1), foram observados dois pacientes com desvio (angulação progressiva maior que $3^{\circ}$ ). Um dos pacientes cujas radiografias mostraram desvio apresentou leve afundamento da placa terminal da vértebra superior devido à 


\section{TABELA 1 - Pacientes com controle de alinhamento da artrodese cervical}

\begin{tabular}{|c|c|c|c|c|c|c|c|}
\hline \multicolumn{4}{|c|}{ Grupo de enxerto ilíaco } & \multicolumn{4}{|c|}{ Grupo de enxerto em bloco hidroxiapatita } \\
\hline Identificação & Sexo / idade & Ângulo POi* & Ângulo final & Identificação & Sexo / idade & Ângulo POi* & Ângulo final \\
\hline 1-A.T.R. & ㅇ/ $26 a$ & $5^{\circ}$ & $5^{\circ}$ & 1-E.A.R. & $9 / 49 a$ & $12^{\circ}$ & $12^{\circ}$ \\
\hline 2-C.G.C. & $\pi / 50 a$ & $5^{\circ}$ & $4^{\circ}$ & 2- A.M.M. & $\pi / 37 a$ & $3^{\circ}$ & $5^{\circ}$ \\
\hline 3- E.R.F.S. & $+/ 55 a$ & $3^{\circ}$ & $6^{\circ}$ & 3- E.R.O.A. & $q / 50 a$ & $5^{\circ}$ & $6^{\circ}$ \\
\hline 4- E.A. & $\hat{o} / 50 a$ & $3^{\circ}$ & $5^{\circ}$ & 4- E.G.T. & $o / 27 a$ & $1^{\circ}$ & $4^{\circ}$ \\
\hline 5- M.L.K. & $a / 47 a$ & $1^{\circ}$ & $3^{\circ}$ & 5- E.A.P. & + / $46 a$ & $7^{\circ}$ & $8^{\circ}$ \\
\hline 6-M.M.T & $\delta / 34 a$ & $2^{\circ}$ & $6^{\circ}$ & 6- L.C.E. & + / $39 a$ & $1^{\circ}$ & $4^{\circ}$ \\
\hline 7-M.R.S. & $q / 43 a$ & $4^{\circ}$ & $7^{\circ}$ & 7-M.C. & $q / 55 a$ & $0^{\circ}$ & $3^{\circ}$ \\
\hline 8- S.A. & $+9 / 37 a$ & $2^{\circ}$ & $3^{\circ}$ & 8- M.A.S. & $9 / 46 a$ & $8^{\circ}$ & $8^{\circ}$ \\
\hline 9- V.M.P. & $\hat{o} / 45 a$ & $7^{\circ}$ & $7^{\circ}$ & 9- N.D.Jr & $q / 47 a$ & $1^{\circ}$ & $2^{\circ}$ \\
\hline 10- W.A. & $q / 63 a$ & $0^{\circ}$ & $0^{\circ}$ & 10- R.H.G. & $o / 42 a$ & $1^{\circ}$ & $2^{\circ}$ \\
\hline \multicolumn{4}{|c|}{$\begin{array}{l}\text { Casos com desvios: } \\
<3^{\circ}=7 \text { casos }(70 \%) \\
3^{\circ}=3 \text { casos }(30 \%)\end{array}$} & \multicolumn{4}{|c|}{$\begin{array}{l}\text { Casos com desvios: } \\
<3^{\circ}=8 \text { casos }(80 \%) \\
>3^{\circ}=2 \text { casos }(20 \%)\end{array}$} \\
\hline
\end{tabular}

*POi: Pós-operatório imediato.

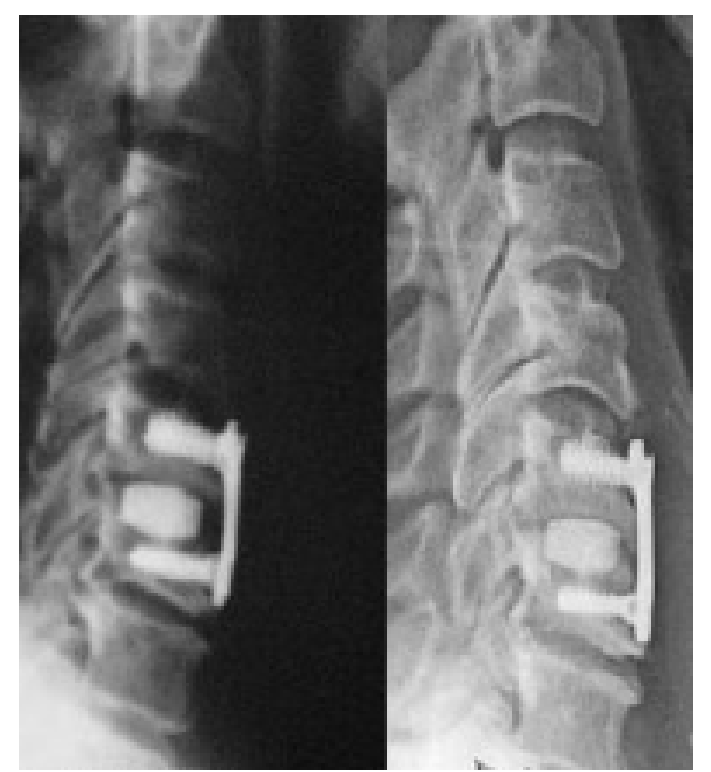

Figura 1

PO 6 semanas (esquerda) e PO 1 ano (direita) de artrodese com bloco de hidroxiapatita

compressão do bloco de hidroxiapatita. Outro paciente apresentou um desvio causado pela osteólise em torno dos parafusos superiores da placa.

Dos dez pacientes operados com utilização de enxerto autólogo bicortical (Figura 2), três pacientes evoluíram com desvio (angulação progressiva maior que $3^{\circ}$ ). Destes, um apresentou desvio devido à má posição do parafuso, associada à fragmentação do enxerto ósseo. Um segundo paciente apresentou perda discreta de altura do enxerto ósseo. O terceiro paciente apresentou fusão espontânea do nível acima ao abordado.

Apesar dos desvios, não houve necessidade de reabordagem cirúrgica em nenhum dos pacientes, uma vez que todos mantiveram bom alinhamento, sem apresentar soltura do material de síntese que necessitasse ser retirado.

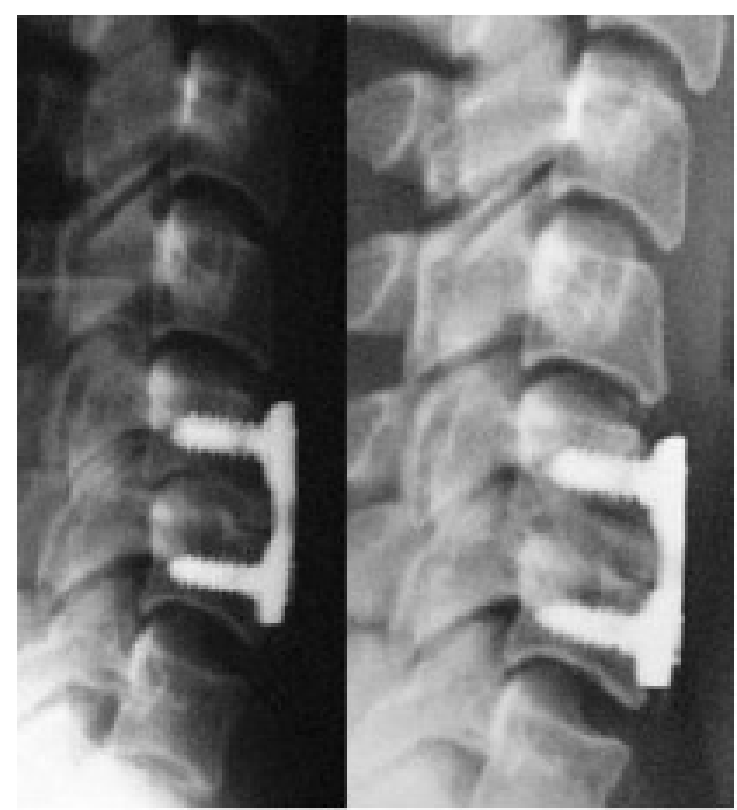

Figura 2

PO 6 semanas (esquerda) e PO 11 meses (direita) de artrodese com enxerto ilíaco

\section{DISCUSSÃO}

Foram avaliados 20 pacientes submetidos à artrodese com placa cervical anterior, sendo utilizado enxerto autólogo em dez pacientes e bloco de hidroxiapatita compacto em outros dez.

Todos os pacientes evoluíram com boa melhora clíni$\mathrm{ca}$, inclusive os que apresentaram desvio maior que $3^{\circ} \mathrm{du}-$ rante o acompanhamento. As complicações resultantes da extração de enxerto foram consideradas menores, da mesma forma como foram consideradas por outros trabalhos ${ }^{12}$, com dor pós-operatória leve persistindo por uma semana em três dos pacientes. Nenhum paciente evoluiu com infecção ou dano neurológico.

O bloco de hidroxiapatita compacto mostrou-se resistente à carga, não apresentando, em nenhum dos pacientes, 
sinais de fragmentação ou perda de altura. A resistência do bloco permitiu que, em alguns casos, houvesse pequeno afundamento na superfície do corpo vertebral (Figura 3). Isso demonstra a importância de avaliar adequadamente $o$

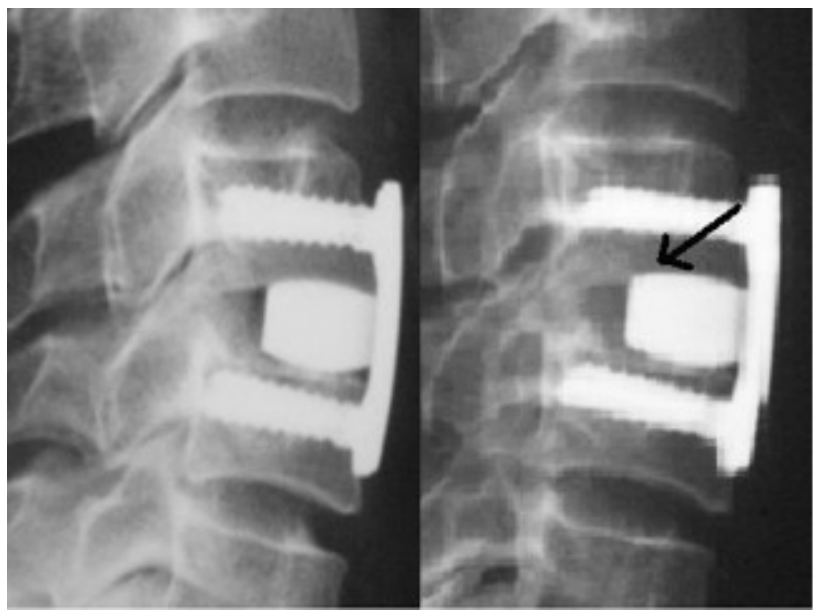

Figura 3

PO imediato (esquerda) e PO 3 meses (direita) de artrodese cervical hidroxiapatita Observar a discordância entre a superfície do corpo vertebral superior e o bloco (seta). tamanho do enxerto, sob o risco de causar perda de alinhamento e deslocamento de enxerto ${ }^{13}$.

Os pacientes submetidos à artrodese com enxerto autólogo mostraram taxas de fusão semelhantes à literatura ${ }^{14}$ $(100 \%)$. No caso dos blocos de hidroxiapatita, não é possível determinar a taxa de osteointegração, pois os blocos são mais radiopacos que o tecido ósseo ${ }^{15}$, dificultando a avaliação da consolidação.

Diversos autores relatam boas taxas de consolidação desse tipo de bloco de hidroxiapatita ${ }^{16,17}$, verificando presença de pontes ósseas em torno do bloco por meio de imagens obtidas por tomografia computadorizada ${ }^{18-21}$. No nosso estudo, não foi realizado o controle por esse método diagnóstico.

O trabalho atual apresenta limitações, como o seu caráter retrospectivo e o pequeno número de pacientes da casuística. Por ser uma série de casos, permite apenas uma estatística descritiva.

\section{CONCLUSÃO}

Nesta pequena série de casos, os resultados da artrodese cervical anterior foram semelhantes em relação à comparação dos grupos com enxerto de hidroxiapatita e com o enxerto autólogo de ilíaco.

\section{REFERÊNCIAS}

1. Cherry C. Anterior cervical discectomy and fusion for cervical disc disease. AORN J. 2002;76(6):998-1004.

2. Nohra G, Abi-Lahoud G, Jabbour P, Salloum C, Rizk T, Samaha E, et al. Anterior cervical discectomy with or without bone graft placement in the treatment of cervical radiculopathy. Long-term results. Neurochirurgie. 2003;49(6):571-8. French.

3. Samartzis D, Shen FH, Matthews DK, Yoon ST, Goldberg EJ, An HS. Comparison of allograft to autograft in multilevel anterior cervical discectomy and fusion with rigid plate fixation. Spine J. 2003;3(6):451-9.

4. McConnell JR, Freeman BJ, Debnath UK, Grevitt MP, Prince HG, Webb JK. A prospective randomized comparison of coralline hydroxyapatite with autograft in cervical interbody fusion. Spine (Phila Pa 1976). 2003;28(4):317-23.

5. Savolainen S, Rinne J, Hernesniemi J. A prospective randomized study of anterior single-level cervical disc operations with long-term followup: surgical fusion is unnecessary. Neurosurgery. 1998;43(1):51-5.
6. Wigfield CC, Nelson RJ.

Nonautologous interbody fusion materials in cervical spine surgery: how strong is the evidence to justify their use? Spine (Phila Pa 1976). 2001;26(6):687-94

7. Resende AC, Cunha LR, Saska S, Balducci-Roslindo E, MinarelliGaspar AM. Análise biológica da hidroxiapatita após implantação em tíbias de ratos. Rev Bras Ortop. 2006;41(4):132-6.

8. Damien E, Hing K, Saeed S, Revell PA. A preliminary study on the enhancement of the osteointegration of a novel synthetic hydroxyapatite scaffold in vivo. J Biomed Mater Res A. 2003;66(2):241-6.

9. De Bôer HH. The History of Bone Graft. Clin Orthop Relat Res. 1988;(226):292-8.

10. Thalgott JS, Fritts K, Giuffre JM, Timlin M. Anterior interbody fusion of the cervical spine with coralline hydroxyapatite. Spine (Phila Pa 1976). 2002;27(24):E518-25; discussion E526-7.

11. Williams KD. Cirurgia ortopédica. Campbell. 10a ed. 2006. p.1696-8.
12. Castro FP Jr, Holt RT, Majd M, Whitecloud TS 3rd. A cost analysis of two anterior cervical fusion procedures. J Spinal Disord. 2000;13(6):511-4.

13.Ito M, Abumi K, Shono Y, Kotani Y, Minami A, Kaneda K. Complications related to hydroxyapatite vertebral spacer in anterior cervical spine surgery. Spine (Phila Pa 1976). 2002;27(4):428-31.

13.Malloy KM, Hilibrand AS. Autograft versus allograft in degenerative cervical disease. Clin Orthop Relat Res. 2002(394):27-38.

14.Floyd T, Ohnmeiss D. A meta-analysis of autograft versus allograft in anterior cervical fusion. Eur Spine J. 2000;9(5):398-403.

15.Senter HJ, Kortyna R, Kemp WR. Anterior cervical discectomy with hydroxyapatite fusion. Neurosurgery. 1989;25(1):39-42.

16. Falavigna A, Righesso O, Volquind D, Teles AR. Anterior cervical interbody fusion with hydroxyapatite graft: clinical and radiological analysis of graft breakage. Spine (Phila Pa 1976). 2009;34(25):2769-74. 
17.Suetsuna F, Yokoyama T, Kenuka E, Harata S. Anterior cervical fusion using porous hydroxyapatite ceramics for cervical disc herniation. A two year follow-up. Spine J. 2001;1(5):348-57.

18.Papavero L, Zwönitzer R, Burkard I, Klose K, Herrmann HD. A composite bone graft substitute for anterior cervical fusion: assessment of osseointegration by quantitative computed tomography. Spine (Phila Pa 1976). 2002;27(10):1037-43.
19. Suchomel P, Barsa P, Buchvald P, Svobodnik A, Vanickova E. Autologous versus allogenic bone grafts in instrumented anterior cervical discectomy and fusion: a prospective study with respect to bone union pattern. Eur Spine J. 2004;13(6);5105.

20.Kim SC, Kang SW, Kim SH, Cho KH, Kim SH. Clinical and radiological outcomes of anterior cervical interbody fusion using hydroxyapatite spacer. J Korean Neurosurg Soc. 2009;46(4):300-4.
21.Iseda T, Nakano S, Suzuki Y, Miyahara D, Uchinokura S, Moriyama T, et al. Radiographic and scintigraphic courses of union in cervical interbody fusion: hydroxyapatite grafts versus iliac bone autografts. J Nucl Med. 2000;41(10):1642-5.

\section{Correspondência}

Yoshinobu Nagasse

Rua da Consolação, 222, conjunto 301, CEP $01302-901$ - São Paulo (SP), Brasil

Fone: 11 3256-6799

E-mail: ynagasse@yahoo.com.br 